\title{
Collisional rates for asymmetrical top molecules ${ }^{\star}$
}

\author{
S. Chandra ${ }^{\star \star}$ and W.H. Kegel \\ Institut für Theoretische Physik der Johann Wolfgang Goethe Universität Frankfurt, Robert-Mayer-Str. 8-10, D-60054 Frankfurt \\ am Main 11, Germany \\ e-mail: kegel@astro.uni-frankfurt.de
}

Received May 10; accepted December 13, 1999

\begin{abstract}
Collisional rate coefficients for rotational transitions in $\mathrm{C}_{3} \mathrm{H}_{2}$ and $\mathrm{SiC}_{2}$ due to collisions with $\mathrm{H}_{2}$ molecules are calculated. The $\mathrm{C}_{3} \mathrm{H}_{2}$ has two distinct species, ortho and para, whereas the $\mathrm{SiC}_{2}$ has only ortho specie due to spin statistics for the identical carbon nuclei. For ortho- and para- $\mathrm{C}_{3} \mathrm{H}_{2}$ we accounted for 47 and 48 energy levels, respectively. The calculations are done for the kinetic temperatures 30, 60, 90, and $120 \mathrm{~K}$. For ortho- $\mathrm{SiC}_{2}$, we accounted for 40 energy levels and the calculations are done for the kinetic temperatures 25 , $50,75,100$, and $125 \mathrm{~K}$. The results are reported here for the transitions in the upward direction. The values for the downward direction can easily be calculated with the help of the detailed equilibrium equation.
\end{abstract}

Key words: ISM: interstellar molecules — molecular data

\section{Introduction}

Since in most regions of interstellar space the excitation of molecules (and atoms) deviates strongly from that under LTE conditions, a quantitative interpretation of observed interstellar molecular lines requires the simultaneous solution of the set of rate equations and the radiative transfer equation for a large number of lines. (In the rate equations one has to account for many more lines than are usually observed.) If all lines of the molecule under consideration are optically thin, the problem reduces to the solution of the - then linear - rate equations. The rate equations describe the interplay between radiative and collisional

Send offprint requests to: W.H. Kegel

* Tables 3A, 3B, 3C, 3D, 4A, 4B, 4C, 4D, 5A, 5B, 5C, 5D and $5 \mathrm{E}$ are available only in electronic form via anonymous ftp (130.79.128.5) at the CDS to cdsarc.u-strasbg.fr or via http://cdsweb.u-strabg.fr/Abstract.html

** On leave from the School of Physical Sciences, S.R.T.M. University, Nanded 431 606, India. transitions and the solution yields the relative occupation numbers from which the line intensities follow. As input parameters one has to know the radiative and the collisional transition probabilities. While for purely rotational transitions the radiative transition probabilities are known for many molecules, the collisional rate coefficients present a problem in most cases. Since in interstellar molecular clouds hydrogen is predominantly in the form of $\mathrm{H}_{2}$, one usually considers only collisions with $\mathrm{H}_{2}$ molecules. To reduce the complexity of the problem one accounts in most cases only for $\mathrm{H}_{2}$ molecules in the rotational ground state $(J=0)$. To simplify the problem even further, one often considers collisions with He atoms assuming that the rate coefficients for collisions with $\mathrm{H}_{2}$ may be obtained from the results for $\mathrm{He}$ by rescaling the latter accounting for the difference of the reduced mass. - In actual NLTE calculations one has to account for a large number of energy levels, which is possible only if the corresponding collisional rate coefficients are known. The goal of such computations is to determine the physical parameters in molecular clouds, in particular the temperature and the density. This requires that the rate coefficients are known for a sufficiently large range of temperatures.

The calculation of the collisional rate coefficients is a difficult task, and the situation becomes even more difficult for the case of an asymmetrical top molecule colliding with $\mathrm{H}_{2}$ molecules. In the present investigation, we have accounted for the collisional transitions between the rotational energy levels in $\mathrm{C}_{3} \mathrm{H}_{2}$ and $\mathrm{SiC}_{2}$ molecules, extending previous work by Green et al. (1987) and Palma \& Green (1987).

\section{Wave-functions for an asymmetrical top molecule}

In an asymmetrical top molecule, all the three moments of inertia about the three principal inertial axes, $a, b$ and $c$, are unequal to one another. The axes are designated as $a$, $b$ and $c$ so that the moments of inertia about them satisfy the condition: $I_{a}<I_{b}<I_{c}$. Obviously, an asymmetrical 
Table 1. A) Wave-functions for ortho levels of $\mathrm{C}_{3} \mathrm{H}_{2}$

\begin{tabular}{|c|c|c|c|c|c|c|c|c|c|}
\hline No. & Level & $E\left(\mathrm{~cm}^{-1}\right)$ & $\epsilon_{J \tau}$ & $g_{J \tau}^{1}$ & $g_{J \tau}^{3}$ & $g_{J \tau}^{5}$ & $g_{J \tau}^{7}$ & $g_{J \tau}^{9}$ & $g_{J \tau}^{11}$ \\
\hline 1 & $1_{01}$ & 1.633 & - & -0.707107 & & & & & \\
\hline 2 & $1_{10}$ & 2.245 & + & 0.707107 & & & & & \\
\hline 3 & $2_{12}$ & 4.480 & - & -0.707107 & & & & & \\
\hline 4 & $2_{21}$ & 6.315 & + & 0.707107 & & & & & \\
\hline 5 & $3_{03}$ & 8.387 & - & -0.676228 & 0.206677 & & & & \\
\hline 6 & $3_{12}$ & 11.155 & + & 0.483241 & -0.516216 & & & & \\
\hline 7 & $3_{21}$ & 12.626 & - & -0.206677 & -0.676228 & & & & \\
\hline 8 & $4_{14}$ & 13.419 & - & -0.651603 & 0.274615 & & & & \\
\hline 9 & $3_{30}$ & 13.530 & + & 0.516216 & 0.483241 & & & & \\
\hline 10 & $4_{23}$ & 17.347 & + & 0.426209 & -0.564221 & & & & \\
\hline 11 & $5_{05}$ & 19.568 & - & -0.629935 & 0.316322 & -0.055880 & & & \\
\hline 12 & $4_{32}$ & 20.204 & - & -0.274615 & -0.651603 & & & & \\
\hline 13 & $4_{41}$ & 22.394 & + & 0.564221 & 0.426209 & & & & \\
\hline 14 & $5_{14}$ & 24.616 & + & 0.371915 & -0.574031 & 0.179351 & & & \\
\hline 15 & $6_{16}$ & 26.834 & - & -0.611098 & 0.343724 & -0.091722 & & & \\
\hline 16 & $5_{23}$ & 28.511 & - & -0.291406 & -0.511000 & 0.392380 & & & \\
\hline 17 & $5_{32}$ & 31.083 & + & 0.344116 & 0.030181 & -0.616987 & & & \\
\hline 18 & $5_{41}$ & 32.489 & - & -0.135148 & -0.372585 & -0.585590 & & & \\
\hline 19 & $6_{25}$ & 33.006 & + & 0.331431 & -0.567582 & 0.260776 & & & \\
\hline 20 & $5_{50}$ & 34.045 & + & 0.493217 & 0.411797 & 0.295229 & & & \\
\hline 21 & $7_{07}$ & 35.217 & - & -0.594594 & 0.362415 & -0.121998 & 0.015160 & & \\
\hline 22 & $6_{34}$ & 38.044 & - & -0.309498 & -0.423776 & 0.473946 & & & \\
\hline 23 & 643 & 41.962 & + & 0.366852 & -0.062054 & -0.601306 & & & \\
\hline 24 & $7_{16}$ & 42.513 & + & 0.299686 & -0.553473 & 0.317154 & -0.057179 & & \\
\hline 25 & $8_{18}$ & 44.717 & - & -0.580000 & 0.375444 & -0.147684 & 0.028826 & & \\
\hline 26 & $6_{52}$ & 44.976 & - & -0.175415 & -0.449741 & -0.516684 & & & \\
\hline 27 & $6_{61}$ & 47.731 & + & 0.505543 & 0.417133 & 0.265380 & & & \\
\hline 28 & $7_{25}$ & 48.675 & - & -0.318245 & -0.345014 & 0.507768 & -0.147840 & & \\
\hline 29 & $8_{27}$ & 53.136 & + & 0.274154 & -0.536222 & 0.356831 & -0.099888 & & \\
\hline 30 & $7_{34}$ & 53.688 & + & 0.336546 & -0.148211 & -0.521837 & 0.304065 & & \\
\hline 31 & $9_{09}$ & 55.334 & - & -0.566976 & 0.384631 & -0.169517 & 0.042949 & -0.004117 & \\
\hline 32 & $7_{43}$ & 57.440 & - & -0.177015 & -0.387188 & -0.223015 & 0.518667 & & \\
\hline 33 & $7_{52}$ & 59.706 & + & 0.261749 & 0.082995 & -0.213490 & -0.615647 & & \\
\hline 34 & $8_{36}$ & 60.423 & - & -0.323060 & -0.279038 & 0.514355 & -0.230670 & & \\
\hline 35 & $7_{61}$ & 61.383 & - & -0.117659 & -0.315762 & -0.421369 & -0.457054 & & \\
\hline 36 & $7_{70}$ & 63.933 & + & 0.477925 & 0.405972 & 0.285519 & 0.158914 & & \\
\hline 37 & $9_{18}$ & 64.877 & + & 0.253141 & -0.517977 & 0.384735 & -0.138890 & 0.017558 & \\
\hline 38 & 845 & 66.571 & + & 0.314342 & -0.208485 & -0.442017 & 0.402920 & & \\
\hline 39 & $10_{1,10}$ & 67.068 & - & -0.555254 & 0.391121 & -0.188132 & 0.056975 & -0.008766 & \\
\hline 40 & 854 & 71.575 & - & -0.202218 & -0.391675 & -0.095246 & 0.544634 & & \\
\hline 41 & $9_{27}$ & 73.287 & - & -0.325373 & -0.223583 & 0.505360 & -0.293416 & 0.051595 & \\
\hline 42 & $8_{63}$ & 75.524 & + & 0.319063 & 0.063310 & -0.303806 & -0.549447 & & \\
\hline 43 & $10_{29}$ & 77.734 & + & 0.235518 & -0.499801 & 0.404154 & -0.173678 & 0.035017 & \\
\hline 44 & $8_{72}$ & 78.873 & - & -0.135426 & -0.357449 & -0.452279 & -0.386437 & & \\
\hline 45 & $11_{0,11}$ & 79.919 & - & -0.544627 & 0.395670 & -0.204065 & 0.070554 & -0.014334 & 0.001118 \\
\hline 46 & $9_{36}$ & 80.560 & + & 0.293570 & -0.250434 & -0.360834 & 0.454239 & -0.120688 & \\
\hline 47 & 881 & 82.372 & + & 0.473527 & 0.406192 & 0.291538 & 0.160579 & & \\
\hline
\end{tabular}

top molecule has no preferential axis, and therefore the treatment of the molecule can be done in six different representations (Chandra 1987). The rotational energy levels in an asymmetrical top molecule may be represented by $J_{\tau}$ or $J_{k_{a}, k_{c}}$, where $\tau, k_{a}$ and $k_{c}$ are sub-quantum numbers and are related through the relations:

$\tau=k_{a}-k_{c}$ and

$$
k_{a}=\operatorname{Int}\left[\frac{J+\tau+1}{2}\right] \quad k_{c}=\operatorname{Int}\left[\frac{J-\tau+1}{2}\right] .
$$

Thus, $\tau$ can assume an integer value ranging from $-J$ to $+J$. However, $k_{a}$ and $k_{c}$ can, independently, assume positive integer values ranging from 0 to $J$. In case of a planar asymmetrical top molecule, the electric dipole of the molecule lies in the $a b$-plane. When the electric dipole 
Table 1. B) Wave-functions for ortho levels of $\mathrm{C}_{3} \mathrm{H}_{2}$

\begin{tabular}{|c|c|c|c|c|c|c|c|c|c|}
\hline No. & Level & $E\left(\mathrm{~cm}^{-1}\right)$ & $\epsilon_{J \tau}$ & $g_{J \tau}^{0}$ & $g_{J \tau}^{2}$ & $g_{J \tau}^{4}$ & $g_{J \tau}^{6}$ & $g_{J \tau}^{8}$ & $g_{J \tau}^{10}$ \\
\hline 1 & $0_{00}$ & 0.000 & + & 1.000000 & & & & & \\
\hline 2 & $1_{11}$ & 1.729 & + & 1.000000 & & & & & \\
\hline 3 & $2_{02}$ & 4.468 & + & 0.827119 & -0.397413 & & & & \\
\hline 4 & $2_{11}$ & 6.027 & - & 0.000000 & -0.707107 & & & & \\
\hline 5 & $2_{20}$ & 6.747 & + & 0.562027 & 0.584861 & & & & \\
\hline 6 & $3_{13}$ & 8.389 & + & 0.766296 & -0.454307 & & & & \\
\hline 7 & $3_{22}$ & 11.215 & - & 0.000000 & -0.707107 & & & & \\
\hline 8 & $3_{31}$ & 13.201 & + & 0.642488 & 0.541853 & & & & \\
\hline 9 & $4_{04}$ & 13.419 & + & 0.718896 & -0.479649 & 0.107382 & & & \\
\hline 10 & $4_{13}$ & 17.340 & - & 0.000000 & -0.635216 & 0.310645 & & & \\
\hline 11 & $5_{15}$ & 19.568 & + & 0.683173 & -0.490823 & 0.160406 & & & \\
\hline 12 & $4_{22}$ & 20.032 & + & 0.470746 & 0.222275 & -0.582917 & & & \\
\hline 13 & $4_{31}$ & 21.441 & - & 0.000000 & -0.310645 & -0.635216 & & & \\
\hline 14 & 440 & 22.621 & + & 0.511455 & 0.469606 & 0.385586 & & & \\
\hline 15 & $5_{24}$ & 24.617 & - & 0.000000 & -0.584861 & 0.397413 & & & \\
\hline 16 & $6_{06}$ & 26.834 & + & 0.654761 & -0.494988 & 0.199460 & -0.029101 & & \\
\hline 17 & $5_{33}$ & 28.538 & + & 0.487527 & 0.143014 & -0.600588 & & & \\
\hline 18 & $5_{42}$ & 31.454 & - & 0.000000 & -0.397413 & -0.584861 & & & \\
\hline 19 & $6_{15}$ & 33.006 & - & 0.000000 & -0.539835 & 0.445198 & -0.101869 & & \\
\hline 20 & $5_{51}$ & 33.902 & + & 0.543684 & 0.488507 & 0.336993 & & & \\
\hline 21 & $7_{17}$ & 35.217 & + & 0.631379 & -0.495423 & 0.229268 & -0.051692 & & \\
\hline 22 & $6_{24}$ & 38.042 & + & 0.467641 & 0.064532 & -0.571815 & 0.243966 & & \\
\hline 23 & $6_{33}$ & 41.885 & - & 0.000000 & -0.390129 & -0.367525 & 0.461221 & & \\
\hline 24 & $7_{26}$ & 42.513 & - & 0.000000 & -0.501251 & 0.471081 & -0.163799 & & \\
\hline 25 & $6_{42}$ & 44.304 & + & 0.325435 & 0.207486 & -0.110560 & -0.625917 & & \\
\hline 26 & $8_{08}$ & 44.717 & + & 0.611633 & -0.493777 & 0.252481 & -0.072993 & 0.007900 & \\
\hline 27 & $6_{51}$ & 45.793 & - & 0.000000 & -0.237439 & -0.408319 & -0.52621 & & \\
\hline 28 & $6_{60}$ & 47.814 & + & 0.496682 & 0.455820 & 0.347879 & 0.218773 & & \\
\hline 29 & $7_{35}$ & 48.675 & + & 0.452248 & 0.007015 & -0.530786 & 0.340519 & & \\
\hline 30 & 817 & 53.136 & - & 0.000000 & -0.468041 & 0.483902 & -0.213926 & 0.031800 & \\
\hline 31 & $7_{44}$ & 53.698 & - & 0.000000 & -0.407106 & -0.252299 & 0.520202 & & \\
\hline 32 & $9_{19}$ & 55.334 & + & 0.594622 & -0.490941 & 0.270828 & -0.092671 & 0.015947 & \\
\hline 33 & $7_{53}$ & 57.623 & + & 0.386067 & 0.211407 & -0.205920 & -0.581705 & & \\
\hline 34 & $8_{26}$ & 60.423 & + & 0.438239 & -0.036789 & -0.484223 & 0.400509 & -0.087982 & \\
\hline 35 & $7_{62}$ & 60.778 & - & 0.000000 & -0.288119 & -0.463064 & -0.450066 & & \\
\hline 36 & $7_{71}$ & 63.886 & + & 0.497780 & 0.458054 & 0.351140 & 0.207351 & & \\
\hline 37 & $9_{28}$ & 64.877 & - & 0.000000 & -0.439270 & 0.488639 & -0.254398 & 0.059624 & \\
\hline 38 & 835 & 66.570 & - & 0.000000 & -0.405374 & -0.146845 & 0.525963 & -0.193574 & \\
\hline 39 & $10_{0,10}$ & 67.068 & + & 0.579734 & -0.487425 & 0.285498 & -0.110612 & 0.024943 & -0.002146 \\
\hline 40 & 844 & 71.545 & + & 0.370380 & 0.158669 & -0.278315 & -0.445159 & 0.361396 & \\
\hline 41 & $9_{37}$ & 73.287 & + & 0.425919 & -0.070707 & -0.437671 & 0.436124 & -0.150122 & \\
\hline 42 & $8_{53}$ & 75.155 & - & 0.000000 & -0.261843 & -0.328501 & -0.0866310 & 0.562157 & \\
\hline 43 & $8_{62}$ & 77.309 & + & 0.262033 & 0.181832 & -0.026777 & -0.288719 & -0.590365 & \\
\hline 44 & $10_{19}$ & 77.734 & - & 0.000000 & -0.414146 & 0.488263 & -0.286888 & 0.087661 & -0.009638 \\
\hline 45 & $8_{71}$ & 79.290 & - & 0.000000 & -0.219197 & -0.369272 & -0.412424 & -0.381442 & \\
\hline 46 & $11_{1,11}$ & 79.919 & + & 0.566537 & -0.483534 & 0.297340 & -0.126851 & 0.034461 & -0.004794 \\
\hline 47 & $9_{46}$ & 80.561 & - & 0.000000 & -0.399157 & -0.061842 & 0.502292 & -0.290778 & \\
\hline 48 & 880 & 82.397 & + & 0.477503 & 0.443387 & 0.351575 & 0.229650 & 0.114276 & \\
\hline
\end{tabular}

of the molecule is along the $a$-axis of inertia, the molecule is known as $a$-type asymmetrical top molecule, and when it is along the $b$-axis of inertia, the molecule is said to be $b$ type asymmetrical top molecule. In case the electric dipole lies in between the $a$ and $b$ axes, it can be resolved into two components along the two axes, and such molecule has both $a$ and $b$ type transitions. In an $a$-type asymmetrical top molecule, the rotational energy levels are grouped into two distinct categories:

$\left(k_{a}, k_{c}\right)$ : (odd, even) or (odd, odd) one category (even, even) or (even, odd) another category 
Table 2. Wave-functions for ortho levels of $\mathrm{SiC}_{2}$

\begin{tabular}{|c|c|c|c|c|c|c|c|c|c|}
\hline No. & Level & $E\left(\mathrm{~cm}^{-1}\right)$ & $\epsilon_{J \tau}$ & $g_{J \tau}^{0}$ & $g_{J \tau}^{2}$ & $g_{J \tau}^{4}$ & $g_{J \tau}^{6}$ & $g_{J \tau}^{8}$ & $g_{J \tau}^{10}$ \\
\hline 1 & $0_{00}$ & 0.000 & + & 1.000000 & & & & & \\
\hline 2 & $1_{01}$ & 0.787 & + & 1.000000 & & & & & \\
\hline 3 & $2_{02}$ & 2.357 & + & 0.999583 & 0.020428 & & & & \\
\hline 4 & $3_{03}$ & 4.701 & + & 0.997932 & 0.045452 & & & & \\
\hline 5 & $2_{21}$ & 7.788 & - & 0.000000 & -0.707107 & & & & \\
\hline 6 & $2_{20}$ & 7.793 & + & 0.028889 & -0.706812 & & & & \\
\hline 7 & $4_{04}$ & 7.805 & + & 0.993927 & 0.077808 & 0.000857 & & & \\
\hline 8 & $3_{22}$ & 10.148 & - & 0.000000 & -0.707107 & & & & \\
\hline 9 & $3_{21}$ & 10.171 & + & 0.064278 & -0.705644 & & & & \\
\hline 10 & $5_{05}$ & 11.654 & + & 0.986385 & 0.116259 & 0.002504 & & & \\
\hline 11 & $4_{23}$ & 13.292 & - & 0.000000 & -0.707030 & -0.010411 & & & \\
\hline 12 & $4_{22}$ & 13.359 & + & 0.110043 & -0.702736 & -0.010391 & & & \\
\hline 13 & $6_{06}$ & 16.230 & + & 0.974474 & 0.158655 & 0.005368 & 0.000040 & & \\
\hline 14 & $5_{24}$ & 17.216 & - & 0.000000 & -0.706812 & -0.020428 & & & \\
\hline 15 & $5_{23}$ & 17.370 & + & 0.164448 & -0.697183 & -0.020343 & & & \\
\hline 16 & $7_{07}$ & 21.517 & + & 0.958119 & 0.202263 & 0.009672 & 0.000136 & & \\
\hline 17 & $6_{25}$ & 21.917 & - & 0.000000 & -0.706371 & -0.032245 & -0.000273 & & \\
\hline 18 & $6_{24}$ & 22.218 & + & 0.224483 & -0.688316 & -0.032014 & -0.000273 & & \\
\hline 19 & $7_{26}$ & 27.389 & - & 0.000000 & -0.705615 & -0.045896 & -0.000732 & & \\
\hline 20 & $8_{08}$ & 27.502 & + & 0.938122 & 0.244385 & 0.015471 & 0.000330 & $1.87 \mathrm{D}-06$ & \\
\hline 21 & $7_{25}$ & 27.913 & + & 0.286321 & -0.675978 & -0.045430 & -0.000733 & & \\
\hline 22 & $4_{41}$ & 29.574 & - & 0.000000 & -0.010411 & 0.707030 & & & \\
\hline 23 & $4_{40}$ & 29.574 & + & 0.000413 & -0.010422 & 0.707030 & & & \\
\hline 24 & $5_{42}$ & 33.512 & - & 0.000000 & -0.020428 & 0.706812 & & & \\
\hline 25 & $5_{41}$ & 33.512 & + & 0.001238 & -0.020478 & 0.706810 & & & \\
\hline 26 & $8_{27}$ & 33.629 & - & 0.000000 & -0.704441 & -0.061322 & -0.001491 & $-9.08 \mathrm{D}-06$ & \\
\hline 27 & $9_{09}$ & 34.179 & + & 0.915870 & 0.282980 & 0.022653 & 0.000664 & $6.90 \mathrm{D}-06$ & \\
\hline 28 & $8_{26}$ & 34.463 & + & 0.346182 & -0.660609 & -0.060603 & -0.001503 & $-9.24 \mathrm{D}-06$ & \\
\hline 29 & 643 & 38.240 & - & 0.000000 & -0.032246 & 0.706306 & 0.009593 & & \\
\hline 30 & $6_{42}$ & 38.240 & + & 0.002769 & -0.032403 & 0.706296 & 0.009593 & & \\
\hline 31 & $9_{28}$ & 40.631 & - & 0.000000 & -0.702740 & -0.078416 & -0.002640 & -0.000030 & \\
\hline 32 & $10_{0,10}$ & 41.543 & + & 0.892835 & 0.316956 & 0.030999 & 0.001178 & 0.000018 & $-2.89 \mathrm{D}-08$ \\
\hline 33 & $9_{27}$ & 41.868 & + & 0.401203 & -0.643029 & -0.077622 & -0.002690 & -0.000031 & \\
\hline 34 & $7_{44}$ & 43.761 & - & 0.000000 & -0.045900 & 0.705384 & 0.018054 & & \\
\hline 35 & 743 & 43.761 & + & 0.005302 & -0.046300 & 0.705348 & 0.018054 & & \\
\hline 36 & $10_{29}$ & 48.387 & - & 0.000000 & -0.700406 & -0.097022 & -0.004268 & -0.000072 & $-3.44 \mathrm{D}-07$ \\
\hline 37 & $11_{0,11}$ & 49.594 & + & 0.870196 & 0.346057 & 0.040250 & 0.001905 & 0.000040 & $3.47 \mathrm{D}-07$ \\
\hline 38 & $8_{45}$ & 50.076 & - & 0.000000 & -0.061334 & 0.703903 & 0.027556 & 0.000210 & \\
\hline 39 & $8_{44}$ & 50.078 & + & 0.009185 & -0.062220 & 0.703795 & 0.027554 & 0.000210 & \\
\hline 40 & $10_{28}$ & 50.125 & + & 0.449816 & -0.624074 & -0.096670 & -0.004429 & -0.000076 & $-4.11 \mathrm{D}-07$ \\
\hline
\end{tabular}

whereas in a $b$-type asymmetrical top molecule, the rotational energy levels are grouped into the following two categories:

\section{$\left(k_{a}, k_{c}\right)$ : (even, odd) or (odd, even) one category (even, even) or (odd, odd) another category}

This grouping of energy levels is independent of the aforesaid representations. Spectroscopists generally use $\mathrm{I}^{r}$ representation in which the $a$-axis of inertia is used as the preferential direction (Sharma \& Chandra 1996; Chandra \& Rashmi 1998). However, for calculations of the collisional rate coefficients, the direction of the electric dipole of the molecule is generally taken as the preferential direction (Green et al. 1987; Palma \& Green 1987). Consequently, for collisional transitions in an $a$-type asymmetrical top molecule, one has to use $\mathrm{I}^{r}$ representation, whereas in the $b$-type asymmetrical top molecule, one has to use $\mathrm{II}^{r}$ representation.

Rotational wave-functions for an asymmetric top molecule can be expressed as linear combinations of wave-functions for a symmetric top molecule (Chandra \& Rashmi 1998; Chandra et al. 1984):

$\psi_{J \tau M}(\alpha, \beta, \gamma)=\sqrt{\frac{2 J+1}{8 \pi^{2}}} \sum_{K=-J}^{J} g_{J \tau}^{K} D_{M K}^{J}(\alpha, \beta, \gamma)$ 
Values of the expansion coefficients $g_{J \tau}^{K}$ depend on the representation (Chandra 1987). The $\mathrm{C}_{3} \mathrm{H}_{2}$ is a b-type asymmetrical top molecule, and therefore $\mathrm{II}^{r}$ representation is used. We have accounted for 47 and 48 rotational energy levels for ortho- and para- $\mathrm{C}_{3} \mathrm{H}_{2}$, and for the rotational constants for the molecule, $A=35092.508332 \mathrm{MHz}$, $B=32212.946832 \mathrm{MHz}$, and $C=16749.028632 \mathrm{MHz}$, the wave-functions are given in Tables $1 \mathrm{~A}$ and $1 \mathrm{~B}$, respectively. The $\mathrm{SiC}_{2}$ is an a-type asymmetrical top molecule, and therefore $\mathrm{I}^{r}$ representation is used. For ortho-SiC 2 , we have accounted for 40 rotational energy levels, and for the rotational constants for the molecule, $A=52473.6664 \mathrm{MHz}, B=13158.65426 \mathrm{MHz}$, and $C=10441.61928 \mathrm{MHz}$, the wave-functions for one category are given in Table 2. The wave-functions for the second category of $\mathrm{SiC}_{2}$ are not reported here as these levels do not exist due to the spin statistics for the identical carbon nuclei. The coefficients with negative value of $K$ are related to those with positive value of $K$ through the relation

$g_{J \tau}^{-K}=\epsilon_{J \tau} g_{J \tau}^{K}$

where the value of $\epsilon_{J \tau}$ for the levels is given in the table for wave-functions. Thus, the coefficients with negative value of $K$ can be obtained.

\section{Collisional rate coefficients}

Collisional rate coefficient for the rotational transition $J \tau \rightarrow J^{\prime} \tau^{\prime}$ at kinetic temperature $T$, averaged over the Maxwellian distribution is given by

$$
\begin{array}{r}
C\left(J \tau \rightarrow J^{\prime} \tau^{\prime} \mid T\right)=\left(\frac{8 k T}{\pi \mu}\right)^{1 / 2}\left(\frac{1}{k T}\right)^{2} \int_{0}^{\infty} \sigma\left(J \tau \rightarrow J^{\prime} \tau^{\prime} \mid E\right) \\
E \mathrm{e}^{-E / k T} \mathrm{~d} E
\end{array}
$$

where the cross section $\sigma\left(J \tau \rightarrow J^{\prime} \tau^{\prime} \mid E\right)$ for the transition is given by

$$
\begin{array}{r}
\sigma\left(J \tau \rightarrow J^{\prime} \tau^{\prime} \mid E\right)=\left(2 J^{\prime}+1\right) \sum_{L M M^{\prime}} S\left(J, \tau, J^{\prime}, \tau^{\prime} \mid L, M, M^{\prime}\right) \\
q\left(L, M, M^{\prime} \mid E\right) .
\end{array}
$$

Here, the sum is finite, limited by triangle inequalities on $J, J^{\prime}$ and $\mathrm{L}$, and each of $M$ and $M^{\prime}$ can independently assume the values ranging from $-L$ to $+L$. The interaction potential is expressed in terms of spherical harmonics, and therefore, the scattering cross section is expressed here in terms of a parameter $q\left(L, M, M^{\prime} \mid E\right)$ (Hutson \& Green 1995). The spectroscopic coefficients, $S\left(J, \tau, J^{\prime}, \tau^{\prime} \mid L, M, M^{\prime}\right)$, depend on the wave-functions of the molecule and on angular momentum coupling factors:

$$
\begin{aligned}
S\left(J, \tau, J^{\prime}, \tau^{\prime} \mid L, M, M^{\prime}\right)= & \sum_{p, p^{\prime}, q, q^{\prime}} g_{J \tau}^{p} g_{J \tau}^{q} g_{J^{\prime} \tau^{\prime}}^{p^{\prime}} g_{J^{\prime} \tau^{\prime}}^{q^{\prime}}(-1)^{p^{\prime}+q^{\prime}} \\
& \left(\begin{array}{ccc}
J & L & J^{\prime} \\
-p & M & p^{\prime}
\end{array}\right)\left(\begin{array}{ccc}
J & L & J^{\prime} \\
-q & M^{\prime} & q^{\prime}
\end{array}\right) .
\end{aligned}
$$

Table 3. A) Collisional rate coefficient $\left(\mathrm{cm}^{3} \mathrm{~s}^{-1}\right)$ for transitions in ortho-C3H2 at $30 \mathrm{~K}$

\begin{tabular}{|cccc|}
\hline Transition & Rate & $l$ & $u$ \\
\hline $1(0,1)==>1(1,0)$ & $7.800 \mathrm{D}-12$ & 1 & 2 \\
$1(0,1)==>2(1,2)$ & $3.041 \mathrm{D}-11$ & 1 & 3 \\
$1(0,1)==>2(2,1)$ & $1.666 \mathrm{D}-11$ & 1 & 4 \\
$1(0,1)==>3(0,3)$ & $3.489 \mathrm{D}-12$ & 1 & 5 \\
$1(1,0)==>2(1,2)$ & $2.409 \mathrm{D}-12$ & 2 & 3 \\
$1(1,0)==>2(2,1)$ & $1.143 \mathrm{D}-11$ & 2 & 4 \\
$1(1,0)==>3(0,3)$ & $4.431 \mathrm{D}-11$ & 2 & 5 \\
$2(1,2)==>2(2,1)$ & $1.217 \mathrm{D}-11$ & 3 & 4 \\
$2(1,2)==>3(0,3)$ & $9.661 \mathrm{D}-12$ & 3 & 5 \\
\hline
\end{tabular}

These spectroscopic coefficients are obviously independent of collision dynamics. Thus, the rate coefficient is given by

$$
\begin{array}{r}
C\left(J \tau \rightarrow J^{\prime} \tau^{\prime} \mid E\right)=\left(2 J^{\prime}+1\right) \sum_{L M M^{\prime}} S\left(J, \tau, J^{\prime}, \tau^{\prime} \mid L, M, M^{\prime}\right) \\
Q\left(L, M, M^{\prime} \mid T\right)
\end{array}
$$

where

$$
\begin{array}{r}
Q\left(L, M, M^{\prime} \mid T\right)=\left(\frac{8 k T}{\pi \mu}\right)^{1 / 2}\left(\frac{1}{k T}\right)^{2} \int_{0}^{\infty} q\left(L, M, M^{\prime} \mid E\right) \\
E \mathrm{e}^{-E / k T} \mathrm{~d} E .
\end{array}
$$

Now, owing to symmetries of $g_{J \tau}^{K}, S\left(L, M, M^{\prime}\right)=S(L$, $\left.M^{\prime}, M\right)$, so that only the real part of $Q\left(L, M, M^{\prime}\right)$ is required, and the cross sections are real. - Green et al. (1987) calculated the $Q\left(L, M, M^{\prime} \mid T\right)$ for $\mathrm{C}_{3} \mathrm{H}_{2}$. These calculations were based on a potential energy surface describing the interaction of $\mathrm{C}_{3} \mathrm{H}_{2}$ with $\mathrm{He}$ and used the infinite order sudden approximation (IOSA) according to Green (1979). They derived the $Q\left(L, M, M^{\prime} \mid T\right)$ for $L \leq 25, M^{\prime} \leq M \leq 8$ and $T=30,60,90$, and $120 \mathrm{~K}$. Despite the larger parameter space for which the $Q\left(L, M, M^{\prime} \mid T\right)$ were calculated, Green et al. give the state-to-state rate coefficients only for $T=60 \mathrm{~K}$ and for transitions between the lowest 20 energy levels of ortho- $\mathrm{C}_{3} \mathrm{H}_{2}$ and the lowest 22 of para- $\mathrm{C}_{3} \mathrm{H}_{2}$. - Similar calculations were performed by Palma \& Green (1987) for $\mathrm{SiC}_{2}$. They computed the $Q\left(L, M, M^{\prime} \mid T\right)$ for $L \leq 25, M^{\prime} \leq M \leq 6$ and $T=25,50,75,100$, and $125 \mathrm{~K}$. State-to-state coefficients were given only for $T=100 \mathrm{~K}$ and for transitions between the lowest 18 energy levels.

As mentioned in the introduction, in astrophysical applications, i.e. for actual NLTE calculations, one needs to know the state-to-state rate coefficients for a large number of transitions in a sufficiently large range of temperatures. We therefore extended the work of Green et al. and Palma and Green, respectively, by using the $Q\left(L, M, M^{\prime} \mid T\right)$ computed by them to calculate state-to-state rate coefficients for an extended set of transitions and for all temperatures for which the $Q\left(L, M, M^{\prime} \mid T\right)$ were given. 


\section{Results}

The calculations are made for the upward transitions $J \tau \rightarrow J^{\prime} \tau^{\prime}$. For ortho- $\mathrm{C}_{3} \mathrm{H}_{2}$, we accounted for 47 energy levels given in Table $1 \mathrm{~A}$. The calculations are done for the kinetic temperatures $30,60,90$, and $120 \mathrm{~K}$, and the results are presented in Tables $3 \mathrm{~A}, 3 \mathrm{~B}, 3 \mathrm{C}$, and $3 \mathrm{D}$, respectively. For para $-\mathrm{C}_{3} \mathrm{H}_{2}$, we accounted for 48 energy levels given in Table 1B. For this case also, the calculations are done for the kinetic temperatures 30, 60, 90, and $120 \mathrm{~K}$, and the results are presented in Tables $4 \mathrm{~A}, 4 \mathrm{~B}, 4 \mathrm{C}$, and $4 \mathrm{D}$, respectively. For ortho- $\mathrm{SiC}_{2}$, we accounted for 40 energy levels given in Table 2. The calculations are done for the kinetic temperatures 25, 50, 75, 100, and $125 \mathrm{~K}$, and the results are presented in Tables $5 \mathrm{~A}, 5 \mathrm{~B}, 5 \mathrm{C}, 5 \mathrm{D}$, and $5 \mathrm{E}$, respectively. The rate coefficient for the corresponding downward transition $J^{\prime} \tau^{\prime} \rightarrow J \tau$ can easily be calculated with the help of the detailed equilibrium equation

$$
\begin{array}{r}
C\left(J^{\prime} \tau^{\prime} \rightarrow J \tau\right)=\frac{2 J+1}{2 J^{\prime}+1} \exp \left[\frac{h c\left[E\left(J^{\prime} \tau^{\prime}\right)-E(J \tau)\right]}{k T}\right] \\
C\left(J \tau \rightarrow J^{\prime} \tau^{\prime}\right)
\end{array}
$$

where the energy $E$ of the energy level is in $\mathrm{cm}^{-1}$. The Tables 3A, 3B, 3C, 3D, 4A, 4B, 4C, 4D, 5A, 5B, 5C, 5D and $5 \mathrm{E}$ are available only in electronic form via anonymous ftp. However, a part of the Table $3 \mathrm{~A}$ is given in printed form in order to have the information about the content of the tables. There we have given (i) transition, (ii) collisional rate coefficient (in $\mathrm{cm}^{3} \mathrm{~s}^{-1}$ ), (iii) lower energy level number $(l)$ of the transition (according to the table of wave-functions), and (iv) upper energy level number $(u)$ of the transition.

Acknowledgements. This work was done during the visit of Prof. S. Chandra under the scientific exchange program between the INSA, New Delhi (India) and the DFG, Bonn (Germany). Prof. Chandra is thankful to Prof. J.M. Waghmare, Vice-Chancellor of the S.R.T.M. University, Nanded for his encouragements.

\section{References}

Chandra S., 1987, Ap\&SS 138, 221

Chandra S., Varshalovich D.A., Kegel W.H., 1984, A\&AS 55, 51

Chandra S., Rashmi, 1998, A\&AS 131, 137

Green S., 1979, J. Chem. Phys. 70, 816

Green S., DeFrees D.J., McLean A.D., 1987, ApJS 65, 175

Hutson J.M., Green S., 1995, MOLSCAT Version 14 distributed by Collaborative Computational Project 6, Daresbury Laboratory: UK Engineering and Physical Sciences Research Council

Palma A., Green S., 1987, ApJ 316, 830

Sharma A.K., Chandra S., 1996, J. Ap. Astr. 17, 41 